\title{
Resource Allocation in Distributed MIMO Radar for Target Tracking
}

\author{
Xiyu Song ${ }^{1, a}$, Nae Zheng ${ }^{2, b}$ and Liuyang $\mathrm{Gao}^{3, \mathrm{c}}$ \\ ${ }^{1}$ Zhengzhou Information Science and Technology Institute, Zhengzhou, China \\ ${ }^{2}$ Zhengzhou Information Science and Technology Institute, Zhengzhou, China \\ ${ }^{3}$ Zhengzhou Information Science and Technology Institute, Zhengzhou, China \\ a1078018194@qq.com, ${ }^{\mathrm{b}} 13837122426 @ 163 . c o m,{ }^{\mathrm{c}} 275609484 @ q q . c o m$
}

\begin{abstract}
Keywords: Distributed MIMO radar, Target tracking, Resource allocation
Abstract. As an important part of the battlefield defense system, the resource optimization strategy of radar systems is becoming a hot topic. In this paper, for the distributed resource allocation problem in MIMO radar target tracking, starting from the existing research results, we discuss the advantages of distributed MIMO radar, its target tracking algorithms and resource allocation strategies, focus on the resource optimization strategies for the increasing accuracy of distributed MIMO radar target tracking, and make prospects for further research.
\end{abstract}

\section{Introduction}

MIMO radar is experiencing a new concept of the rapid development of radar system, which has incomparable advantages over traditional radars in accurate positioning, low-speed moving target detection, spatial resolution, as well as to deal with stealth targets and low probability of intercept radars and other aspects. The development status and issues to be studied are of concern. Since the communication MIMO technology achieved innovation in the field of radars, the distributed MIMO radar has becoming a major breakthrough of traditional radar concept. For the development of radar technology, the distributed MIMO radar is opening up broader prospects for further development and applications.

In a hostile environment, the resource allocation strategy [1] is an important part in military action, so it must be considered in radar system design. The resource allocation problem is not only a hot topic in this field, but also supports the future of networked radar development. For example, in MIMO radar system design, the optimal power allocation strategy in most cases is not necessarily the uniform power distribution, therefore the power allocation must be considered in resource allocation. The goal of resource allocation is to find an optimal strategy, so that within certain transmission resource limit, parameter estimation error is minimized, or resources consumption within the given parameter estimation error limit to a minimum.

The paper analyses the current situation of "resource allocation in distributed MIMO radar for target tracking", trying to have a fundamental and comprehensive understanding and awareness of the field. The optimal allocation strategies to meet the tracking performance of different motion states in distributed MIMO radar are discussed. In this paper, the existing research results are classified and summarized, research status is described from three aspects, the advantages of distributed MIMO radar, its target tracking algorithms and resource allocation strategies. Finally, combining research problems and deficiencies, scientific questions for further study are pointed out.

\section{The Advantages of Distributed MIMO Radar}

Distributed MIMO radar system with a plurality of uncorrelated transmit and receive channels, whose transmit and receive antennas are widely spaced, can illuminate the target from different angles, collect the real-time data carrying different amplitude, delay or phase information of the target echo data, using space diversity target detection, better spatial resolution, multi-angle estimation and target processing capability are developed. 
Distributed MIMO radar is the continuation of concept of multi-station radar and netted radar [2], the significance of the study is the formation of the signal level in the converged network. The traditional netted radar, which fuses target traces of points and navigation and achieve network detection [3], without using echo data, is belong to information fusion network. However, the echo signal converged network combining data from all receive paths completes the fusion process at a signal level to raise awareness of the radar target detection and performance of parameter estimation.

The ability for information in spatial parallel multi-channel in distributed MIMO radar is a fundamental advantage [4].

One is the design and optimization of the system structure, such as the arrangements for the number and layout of the antenna array elements, to respond to the needs of different tasks or scenes. The other is flexible signal design and processing capabilities, such as the design and configuration of the transmit waveform and signal parameters for better system performance. From a system perspective, transmit parameters, including array element, power, waveform, frequency, bandwidth, and time [5], can be regarded as system resources. Therefore, all of these can be attributed to the resource allocation problem. The rationality of the resource allocation strategies is directly related to that whether the distributed MIMO radar system can maximize performance or not.

\section{Target Tracking Algorithms Based on Distributed MIMO Radar}

The task of target tracking is to associate and filter the measured value from the target, and to predict the next time's parameters to maintain accurate real-time estimation of target motion states. The target tracking algorithm greatly affects the ultimate target tracking accuracy. From the perspective of resource allocation. When the target is of uniform motion or low acceleration motion, resource optimization allocation on target location accuracy is the focus of attention. When the acceleration can't be ignored, velocity estimation accuracy also need to be considered. It is necessary to study the resource optimization allocation to improve the position and velocity estimation precision, to enhance all the parameters estimation precision in the radar system [6].

The key issue to achieve maneuvering target tracking precisely is to make the target model created match the actual target model. The vast majority of traditional target tracking algorithms are based on the Kalman filter theory. However, Kalman filter theory is only applicable to linear systems, and requires that observation equations must be linear. In fact, the actual target tracking process is nonlinear. Thus there are a series of nonlinear filtering algorithms proposed in the twentieth century, such as an extended Kalman filter, unscented Kalman filter and particle filter. In order to better solve the target model matching problem between assumptions and the actual movement, more algorithms have appeared in variable dimension filter, input estimation, Interacting Multiple Model (IMM) tracking algorithms and other models. A variable dimensional filtering algorithm proposed in [7] changes the target state dimension to reach model switching by judging a maneuvering target, but reinitialize of the filter will result in greater fluctuation in the filtering error. Input estimation algorithm mentioned in [8] refers the motor acceleration as unknown deterministic input, employ least squares to estimate the value of the maneuvering acceleration and update the target state. Both methods are based on the assumption: the acceleration is constant or slowly varying. In response to the rapid changing acceleration, Shalom proposed IMM algorithm [10,11], using dynamic models with a plurality of different orders and different noise levels respectively estimate the target state. Then, weight the estimation results of different probability models, and regard the weighted result as the ultimate target tracking results, an excellent robustness and stability is showed in the algorithm. Currently, the mainstream algorithm for maneuvering target tracking is IMM algorithm, a compromise between performance and complexity achieved in IMM algorithm, which has been successfully applied to many tracking systems. But its tracking performance largely depends on the model used, and the tracking performance of weak motorized targets and non-motorized targets will be decreased.

Resource allocation algorithms of existing multi-target tracking mode include methods based on the risk cost, based on information theory and a method based on covariance control. Resource 
allocation algorithms based on the risk cost, transform the resource allocation into a mathematical optimization problem, that's to say, a risk cost minimization process. In practice, it isn't easy to solve the problem with such a complex calculation. In fact, different target tracking radars need different accuracy classes, that all depends on the nature of tracking tasks. The idea of covariance control was first proposed by scholars Kalandros to solve the problem for selecting a combination of radar systems. The basic idea is to set a desired tracking accuracy for each target in advance, by tracking control of each target, to promote the tracking error covariance approach presupposed expectations, and ensure that all the targets can be maintained at a desired tracking state.

In practical applications, due to the target movement model is unknown and likely to change over time. So any single motion model is difficult to describe the actual target motion, while the IMM algorithm becomes a natural choice. By introducing a multiple target motion model, the IMM algorithm employs the model probability weighted approach to estimate the state of each model, and achieve the maneuvering target tracking. When it comes to tracking accuracy specifications of radar resource allocation, the IMM algorithm integrated into the covariance controlled algorithm can better solve the resource allocation for maneuvering target tracking.

\section{Resource Allocation Strategies on the Tracking Model}

The concept of resource optimization for system radar applications is significant. How to efficiently use the limited transmit resources, and how to play the best results are the key to excavate the potential of MIMO radar system. The performance of positioning can be enhanced by increasing the number of radars or the transmit power to achieve. But in the actual, full system use may result in an inefficient use of system resources. How to distribute the limited resources and maximize efficiency of the system resources and whole performance is the key issue to be studied.

The existing resource allocation strategies of MIMO radar systems can be divided into two categories, one is based on system structure mentioned in $[13,14]$, the other mentioned in $[15,16]$ is based on the transmit parameters.

Resource Allocation Strategies Based on the Selection and Layout of Antenna Array Element. For the problem of the system structure, the present studies mainly focus on the intelligent selection of antenna array element and reasonable layout of antenna array element position. The problem of radar subset selection was analyzed in $[9,17]$, in order to reduce the communication connection path associated with the fusion center and signal processing burden, only the partial radar antennas are activated. A heuristic algorithm is proposed, which could select the best radar antenna combination within given antenna limits for the most accurate estimation, or, minimize the total number of antennas within given estimation accuracy requirements. In $[12,18,19,20]$, problems of antenna combination in distributed MIMO radar were modeled as a knapsack problem based on an optimization algorithm, which employed the heuristic algorithms and selected antenna combination, gradually approach the optimal value. The multiple radar grouping strategy for multi-targeting search problem were discussed in [21], which covered different search units to reduce the whole number of radars with the same accuracy threshold. On this basis, a multi-target localization algorithm based on cluster allocation was proposed in [22], whose purpose was to minimize the number of radars, under the premise of meeting the accuracy requirements of each target. The cluster allocation algorithm clusters the radars, according to the number of multi-radar system radar targets, and each target is only tracked by the corresponding subset radars. Thereby, the amount of data transmitted by multi-radar and computational complexity in fusion center is reduced.

Resource Allocation Strategies Based on the transmit parameters. For the problem of the transmit parameters, the studies focus on the transmit power and efficient bandwidth, a power distribution ideal starting from the performance was presented in [15], on the distributed MIMO radar platform. The paper regarded the Cramer Rao Lower Bound (CRLB) of target location error as the power distribution cost function. The purpose was to reasonably distribute limited power resources for the highest location accuracy. Two resource allocation schemes based on location performance for MIMO radar were proposed in $[15,23,24]$. One was to minimize the total transmit energy by 
setting the mean square error threshold for parameter estimation and proposed solutions to this non-convex, non-linear optimization problem by the methods of convex relaxation and domain decomposition, which proved that the uniform power distribution was not necessarily the best choice. The problem of resource allocation was studied for multi-sensor target tracking in the clutter [25], and regarded the posterior Cramer-Rao lower bound (PCRLB) as performance estimation of target state. Considering the uncertainty of source measurement, a scheme was proposed, which could accurately locate the target and effectively use the resources. In [26], a joint scheme of power and bandwidth allocation in the multi-targeting was studied. The simulation results indicated that the impact of the bandwidth allocation strategy was greater than power allocation strategy. On the basis of the distributed MIMO radar and interacting multiple model (IMM) framework, a method of power and bandwidth joint distribution for maneuvering target tracking was proposed in [27]. Using the IMM-EKF algorithm, the resource allocation problem in target tracking is transferred from one resource to a variety of resources optimization, which will be the mainstream for the development of resource optimization.

With game theory [28] widely used in military science and communications, people have begun to apply it to a variety of radar system design and signal processing [29], and modeled for resource optimization problems [30] with game theory. In [31], Non-cooperative game theory was employed to control the transmit power of the radar network, and designed a reasonable target detection threshold and power consumption, according to Nash equilibrium. In [32], the location estimation was modeled as a dynamic cooperative game among the sensors, under the premise of the given number of observations, to assign node number of observations by iterating sharply value and continuously improve the positioning accuracy. Game theory is worthy of further study, which introduced new ideas for resources optimization in distributed MIMO radar.

\section{Conclusion and Outlook}

According to these studies on development of resource optimization based on distributed MIMO radar target tracking, we find distributed MIMO radar technology constantly mature and its huge development potential. However, there are still something faulty to improve on the resource allocation in distributed MIMO radar target tracking:

a. Seek new ideas and methods to optimize the resource allocation problem, instead of limited to the existing optimization theory. The relation between performance evaluation indicator of target tracking and resources to be distributed in radar systems needs to be further improved, so to explore new ideas to solve the problem is necessary. For example, the introduction of game theory to study the resource allocation is a great breakthrough.

b. Adapt to the more complex the battlefield environment. Most of the existing cognitive target tracking algorithms are simply analyzed and simulation in an ideal testing conditions. In practice, the changing RCS of complex targets cannot be ignored, and detection probability is generally less than 1 , and there are a lot of false alarms. For the multi-target case, data association is always a difficulty. To this end, further research is a special need for multi-target recognition tracking algorithms under complex and changing environments.

c. Achieve a joint optimization for multi-resource. For the joint optimization problem of position and velocity estimation in target tracking, present studies are mostly based on one or two aspects of system resources. A variety of resources, including array element, transmit power, the effective bandwidth and time width, could be comprehensively studied. Establishing a complete resource allocation system for distributed MIMO radar target tracking in complex environments is necessary, which will provide a useful theoretical support and technical reference for quickly adapting to the battlefield environment and further practical applications.

Studies on resource allocation in distributed MIMO radar target tracking have great scientific significance for the development of new radar systems. Meanwhile, it also shows potentially broad application prospects for the "slow low small" target detection with a greater value in the theory and application. To fully excavate the potential of MIMO radar system and to expand the specific 
research for the resource allocation in the distributed MIMO radar target tracking tasks is of importance to enrich and improve theoretical connotation of the new radar system.

\section{References}

[1] H.W. Chen. Analysis and Application for MIMO Radar System. Journal of Electronics (2015).

[2] H.W. Chen. in: A New Radar System ——MIMO Radar . Journal of Electronics, Vol.40 (2012), p. 1190-1198

[3] Baumgarten. D: Optimum Detection and Receiver Performance for Multi-static Radar Configurations. Acoustics, Speech, and Signal Processing, IEEE International Conference on ICASSP (1982), p. 359-362

[4] X.M Li: MIMO Radar and Characteristics Survey (Modern Defence Technology, 2015).

[5] J.K Yan. Study on Resource Allocation Scheme for Cognitive Radar. Xi'an University of Electronic Science and Technology (2014).

[6] Doughty, S. R. Development and Performance Evaluation of A Multi-static Radar System. University of London (2008).

[7] Li, X. R, et al. Survey of Maneuvering Target Tracking (2001).

[8] Khaloozadeh H, and Karsaz A. Modified Input Estimation Technique for Tracking Manoeuvring Targets [J]. IET Radar Sonar and Navigation (2009), p.30-41

[9] Gao H, et al. Antenna allocation in MIMO radar with widely separated antennas for multi-target detection. Sensors 14.11(2014), p. 20165-87

[10]Lan, Jian, et al. Second-Order Markov Chain Based Multiple-Model Algorithm for Maneuvering Target Tracking. IEEE Transactions on Aerospace Electronic Systems 49.1(2013), p. 3-19

[11]Laneuville D. and Y. Bar-Shalom. Maneuvering Target Tracking: A Gaussian Mixture Based IMM Estimator. IEEE Aerospace Conference Proceedings 186.6(2012), p. 1-12

[12] Ma Botao, et al. A joint scheme of antenna selection and power allocation for localization in MIMO radar sensor networks. IEEE Communications Letters 18.12(2014), p. 2225-2228

[13]Godrich. H, A. P. Petropulu, and H. V. Poor. Sensor Selection in Distributed Multiple-Radar Architectures for Localization: A Knapsack Problem Formulation. IEEE Transactions on Signal Processing 60.1(2012), p. 247-260

[14] Godrich H, A. Petropulu, and H. V. Poor. Cluster Allocation Schemes for Target Tracking in Multiple Radar Architecture. IEEE (2011), p. 863-867

[15] Godrich H, A. P. Petropulu, and H. V. Poor. Power Allocation Strategies for Target Localization in Distributed Multiple-Radar Architectures. IEEE Transactions on Signal Processing 59.7(2011), p. 3226-3240

[16] Godrich H, A. Petropulu, and H. V. Poor. Resource Allocation Schemes for Target Localization in Distributed Multiple Radar Architectures (2010), p. 1239-1243

[17] Hernandez, M. L, T. Kirubarajan, and Y. Bar-Shalom. Multisensor resource deployment using posterior Cramer-Rao bounds. IEEE Transactions on Aerospace \& Electronic Systems 40.2(2004), p. 399-416

[18] Godrich, Hana, A. P. Petropulu, and H. V. Poor. Sensor Selection in Distributed Multiple-Radar Architectures for Localization: A Knapsack Problem Formulation. IEEE Transactions on Signal Processing 60.1(2012), p. 247-260 
[19] Godrich, Hana, A. Petropulu, and H. V. Poor. A combinatorial optimization framework for subset selection in distributed multiple-radar architectures. IEEE International Conference on Acoustics (2011), p. 2796-2799

[20]Godrich, H, A. Petropulu, and H. V. Poor. Cluster allocation schemes for target tracking in multiple radar architecture. IEEE (2011), p.863-867

[21] Jamali-Rad, Hadi, A. Simonetto, and G. Leus. Sparsity-Aware Sensor Selection: Centralized and Distributed Algorithms. IEEE Signal Processing Letters 21.2(2014), p. 217-220

[22]J.K. Yan. A joint clustering and power allocation algorithm in Multi-Radar System for multi-target tracking [J]. Electronics \& Information Technology (2013), p. 1875-1881

[23]Godrich, H, A. Petropulu, and H. V. Poor. Optimal power allocation in distributed multiple-radar configurations. IEEE International Conference on Acoustics (2011), p. 2492-2495

[24] Godrich, H, A. Petropulu, and H. V. Poor. Power allocation schemes for target localization in widely distributed MIMO radar systems. Military Communications Conference IEEE (2010), p. 846-85

[25] Gorji, A. A, R. Tharmarasa, and T. Kirubarajan. Optimal Antenna Allocation in MIMO Radars with Collocated Antennas. IEEE Transactions on Aerospace \& Electronic Systems 50.1(2015), p. 542-558

[26] Hernandez, M. L, T. Kirubarajan, and Y. Bar-Shalom. Multisensor resource deployment using posterior Cramer-Rao bounds. IEEE Transactions on Aerospace \& Electronic Systems 40.2(2004), p. 399-416

[27]Y.Y. Li. Distributed Resource Allocation algorithm in MIMO Radar for Maneuvering Target Tracking [J]. Xi'an University of Electronic Science and Technology: Natural Science (2015).

[28] Boche, Holger, et al. Game Theory in Signal Processing and Communications. Eurasip Journal on Advances in Signal Processing (2009), p. 1-2

[29] Sad, W. et al. Coalitional game theory for communication networks. IEEE Signal Processing Magazine 26.5(2009), p. 77-97

[30]W. Liu. Resource Allocation Based on Game Theory in Collaboration and sensing networks [D]. Beijing University of Posts (2011).

[31] Panoui, Anastasia, S. Lambotharan, and J. A. Chambers. Game theoretic power allocation technique for a MIMO radar network. International Symposium on Communications, Control and Signal Processing (2014), p. 509-512

[32] Gharehshiran, Omid Namvar, and V. Krishnamurthy. Coalition Formation for Bearings-Only Localization in Sensor Networks-A Cooperative Game Approach. IEEE Transactions on Signal Processing 58.8(2010), p. 4322-4338 